\title{
Camelina sativa: an ideal platform for the metabolic engineering and field production of industrial lipids.
}

\author{
Sunil Bansal ${ }^{1}$ and Timothy P. Durrett ${ }^{1}$ \\ 1Department of Biochemistry and Molecular Biophysics \\ Kansas State University, Manhattan, KS 66506, USA.
}

Corresponding author: Timothy Durrett; email: tdurrett@ksu.edu 


\begin{abstract}
Triacylglycerols (TAG) containing modified fatty acids with functionality beyond those found in commercially grown oil seed crops can be used as feedstocks for biofuels and bio-based materials. Over the years, advances have been made in transgenically engineering the production of various modified fatty acids in the model plant Arabidopsis thaliana. However, the inability to produce large quantities of transgenic seed has limited the functional testing of the modified oil. In contrast, the emerging oil seed crop Camelina sativa possesses important agronomic traits that recommend it as an ideal production platform for biofuels and industrial feedstocks. Camelina possesses low water and fertilizer requirements and is capable of yields comparable to other oil seed crops, particularly under stress conditions. Importantly, its relatively short growing season enables it to be grown as part of a double cropping system. In addition to these valuable agronomic features, Camelina is amenable to rapid metabolic engineering. The development of a simple and effective transformation method, combined with the availability of abundant transcriptomic and genomic data, has allowed the generation of transgenic Camelina lines capable of synthesizing high levels of unusual lipids. In some cases these levels have surpassed what was achieved in Arabidopsis. Further, the ability to use Camelina as a crop production system has allowed for the large scale growth of transgenic oil seed crops, enabling subsequent physical property testing. The application of new techniques such as genome editing will further increase the suitability of Camelina as an ideal platform for the production of biofuels and biomaterials.
\end{abstract}




\section{Introduction}

Dwindling cheap fossil fuel reserves and concerns about climate change have increased the need to obtain fuel and chemical products from renewable sources. While plant oils have long been used as sources of fuel and as feedstocks for industrial chemistry, commercially grown oil seed crops only synthesize a low diversity of fatty acids, limiting the chemical functionality of the oil. However, within the plant kingdom a great diversity of lipids has been found with different structures and fatty acids, many of which confer chemically useful functions[1]. The isolation of enzymes from different plants and an increased understanding of metabolic fluxes in developing seeds have allowed the production of high levels of unusual lipids in transgenic seeds. Until recently, much of this work was pioneered in the model plant Arabidopsis thaliana, which is easily transformed and possesses a great many mutants affecting different aspects of lipid biosynthesis [2, 3]. Not only have these mutants provided insights into the synthesis of fatty acids and subsequent flux into storage lipids, but they have also provided a useful genetic background for the synthesis of unusual fatty acids. However, the small seed yield and lack of large scale field growth options have limited the ability to test the functionality of any transgenic oil produced in Arabidopsis. Instead, insights obtained from work with this model species typically have had to be transferred to oil seed crops. Here we describe the use of the emerging oil seed crop Camelina sativa as an appealing alternative for the production of modified lipids in transgenic plants. We will discuss why Camelina is particularly attractive as an industrial seed crop, both from an agronomic perspective as well as from a seed metabolic engineering system. To illustrate this potential of Camelina, we will describe examples of the metabolic engineering of unusual lipids in this oil seed crop. In particular, we focus on the synthesis of very high levels of acetyl-TAGs in Camelina, the highest accumulation of unusual lipids achieved so far in transgenic seeds. Further, the field growth of these high acetyl-TAG lines allowed the production of large quantities of these unusual lipids, permitting meaningful property testing.

\section{Camelina sativa: an underdeveloped oil seed crop}

Camelina sativa, also known as false flax or gold of pleasure, is a member of the Brassicaceae family. Despite appearing to have been used as an oil seed crop since the Iron Age, Camelina's use waned during the middle ages [4]. However, its various positive agronomic and environmental features, along with the development of tools for genetic manipulation, have contributed to an increased interest of scientists and farmers in Camelina for use as an industrial oilseed crop.

\subsection{Fatty acid profile of Camelina}

Camelina oil contains high proportions of the polyunsaturated fatty acids (PUFAs) linoleate (18:2) ${ }^{1}$ and linolenate (18:3) compared to soybean and canola oil (Figure

\footnotetext{
${ }^{1}$ Fatty acids are represented as $\mathrm{X}: \mathrm{Y}$ where $\mathrm{X}$ indicates the number of carbon atoms and $Y$ represents the number of double bonds. In some situations, the position of double bonds from the carboxyl group is designated by delta $(\Delta)$. Therefore linoleate can be represented as 18:2 or 18:2 $\Delta 9,12$.
} 
1). This high degree of unsaturation renders Camelina oil highly prone to oxidation making it less suitable for certain applications. For example, biodiesel derived from Camelina possesses a lower oil stability index (OSI) compared to biodiesel from other feedstocks [5]. Below we describe successful mutagenic and biotechnology approaches to decrease the PUFA content of Camelina oil to make it more oxidatively stable.

\subsection{Agronomic advantages of Camelina}

Camelina has a number of excellent agronomic properties which make it valuable as an oilseed crop. For example, Camelina has a relatively short growing season (85 to 100 days), and possesses winter and spring variaties, facilitating rotation with other crops [6]. Further, the oil yield of Camelina (variable from 400 to $850 \mathrm{~kg} \mathrm{ha}^{-1}$, depending on the study) is typically comparable with that of Brassica juncea and Brassica rapa and higher than that of soybean [6,7]. While the oil yield is somewhat lower than that of canola, studies have shown that the cost of production of seed oil from Camelina can be less than half that from rapeseed due to comparatively low input requirements [8]. For example, Camelina requires only low amounts of fertilizer, with optimum nitrogen and phosphorus requirements of about 80-100 kg $\mathrm{ha}^{-1}$ and $50 \mathrm{~kg} \mathrm{ha}^{-1}$, respectively $[9,10]$. Increasing the nitrogen content above optimum was found to increase seed yield depending on soil potential and precipitation $[11,12]$. Camelina is also capable of tolerating conditions of water stress and is therefore less dependent on irrigation. For example, in one drought stress study, Camelina possessed a higher seed yield $\left(1383 \mathrm{~kg} \mathrm{~h}^{-1}\right)$ than B. juncea (933 $\mathrm{kg} \mathrm{ha}^{-1}$ ) and Brassica carinata (711 kg ha-1) [13]. Similarly, a two-year study in western Canada showed high drought and flea beetle resistance of Camelina over commercially grown crops such as B. rapa and canola [14]. The evapotranspiration of Camelina was found to be 332 to $371 \mathrm{~mm}$, much lower than that of vegetable and grain crops $(600-655 \mathrm{~mm})$, offering one explanation for the increased tolerance of low water stress [15]. Camelina also appears to be more resistant to diseases like black spot (Alternaria brassicae) and blackleg (Leptosphaeria maculans) which are important pathogens of rapeseed and canola [16-18]. Additionally, some Camelina genotypes are resistant to other common Brassicaceae diseases like sclerotinia stem rot, brown girdling rot and downy mildew [19]. Such germplasm could therefore be used to breed for resistance in other backgrounds.

\subsection{Environmental benefits of Camelina as an industrial oilseed crop.}

Ideally, crops grown for industrial feedstocks or biofuel purposes should not displace other crops grown for food. The agronomic features of Camelina mean that it is well suited for this role. For example, its low water and fertilizer requirements mean it can be cultivated on marginal lands. However, recent work has shown that other alternative biofuel crops, such as Jatropha curcas, require some irrigation for optimal growth and need to be supplemented with fertilizers to produce seed oil if grown on marginal lands [20]. These results suggest that even for crops supposedly suitable for growth on marginal lands, yield under unfavorable conditions without some sort of agricultural input will be low. 
Therefore, instead of trying to grow Camelina on marginal lands, a more effective strategy would be to take advantage of its short generation time and cultivate it on currently used cropland as part of multi crop rotation system. For example, Camelina can be grown as a rotation crop during the fallow years with wheat and dryland cereals, like oats and barley, without affecting the yield of these crops [21]. The same study predicted that the roughly 5-7 million acres of land available through such a fallow rotation would provide a Camelina oil yield of 750 to 1000 million gallons per year. Yield returns of Camelina- soybean and Camelinasunflower were $82 \%$ and $72 \%$ respectively of their monocropped counterparts however the net economic returns for Camelina-soybean double crop were higher than soybean alone [22]. In a subsequent study, winter Camelina grown as double crop and relay crop with soybean showed similar net economic returns compared to the mono-cropped full season soybean [23]. Further, life cycle assessments of biodiesel produced from camelina grown under different conditions, showed reduced energy non-renewable energy inputs and overall green house gas emissions compared to diesel fuel as well as biodiesel from other oilseed crops [24, 25], making Camelina a more environmental friendly substitute than other biofuel crops.

\subsection{Manipulating lipid metabolism in Camelina}

In addition to its useful agronomic traits, the recent development of tools and resources for Camelina have facilitated its emergence as an ideal platform for the metabolic engineering of oil composition and subsequent growth in the field. Importantly, Camelina can be rapidly and easily transformed using agrobacteriummediated floral-dip methods [26, 27]. The development of these methods makes Camelina transformation much simpler and quicker compared to the tissue-culture based approaches required for the transformation of other oilseed crops. Transgenic seeds are easily identified using a variety of selectable markers, including seed fluorescence and resistance to specific herbicides or antibiotics [27]. The existence of multiple selectable markers is useful for stacking different transgenic traits. Additionally, knowledge of the seed transcriptome [28] and the recent sequencing of the genome [29] allow current and future work in Arabidopsis to be easily applied to Camelina. For example, analysis of genomic sequence revealed that over $90 \%$ of the Arabidopsis genes involved in lipid metabolism were also present in the Camelina genome [2, 29]. Similar results were also obtained through analysis of the seed transcriptome, which suggested that approximately $80 \%$ the expressed lipid related genes were $80 \%$ or more identical compared to their Arabidopsis orthologs [28].

\subsubsection{Overcoming the hexaploid genome of Camelina}

One potential drawback to engineering metabolic fluxes in Camelina, is its remarkably undifferentiated hexaploid genome [29]. Therefore, enzymatic activity could be encoded by up to three similar gene homeologues making modifying endogenous metabolic fluxes more difficult. Indeed, earlier work has demonstrated that all three copies of CsFAD2 and CsFAE1 are expressed in developing seeds [30, 
31]. Further, all three CsFAD2 genes encode functional desaturase enzymes based on their ability to synthesize linoleic acid when expressed in yeast [31].

Consequently, RNA interference (RNAi) has been used to facilitate posttranscriptional gene silencing of targeted genes. The high similarity of the three Camelina sub-genomes $[28,29]$ means that all three homeologues can be targeted with a single RNAi construct. Thus RNAi against CsFAD2 was successful in increasing oleic acid levels to around $50 \%$ compared to about $15 \%$ in wild type seeds [28,31]. The simultaneous silencing of both CsFAD2 and CsFAE1 further increased the levels of oleic acid to $70 \%$ in the best lines [28]. As we describe below, other RNAi based strategies have been successfully used to target specific endogenous Camelina activities that compete with the production of a desired lipid.

While RNAi-based approaches have the advantage of targeting all homeologues encoding a particular enzyme activity, in some cases mutagenesis of the genes might be more desirable. For example, the phenotype in a mutant background would be more genetically stable than that achieved through RNAi. Further, some farmers and markets might be more receptive to the adoption of a non-transgenic biofuel crop, such as high-oleic Camelina. Given the hexaploid genome of Camelina, in most cases three separate mutagenic events will be required for complete elimination of enzymatic activity. For example, mutation of the CsFAD2-2 gene increased oleic acid content from $17 \%$ to $27 \%$, lower than the 38 $51 \%$ range observed with RNAi of CsFAD2 [31]. This is not surprising given that more than $80 \%$ of Camelina genes potentially involved in different aspects of acyllipid metabolism were present in three homeologous copies [29]. However, the fact that even a small increase was observed suggests, at least for the case of CsFAD2, that the three genes contribute additively to activity. Further, the presence of phenotype, albeit small, suggests that high throughput screening for mutants with altered fatty acid composition is a viable approach towards obtaining mutations in different homeologues.

The emergence of a number of different genome editing technologies offers new strategies with which to generate mutants in a target gene. The CRISPR/Cas9/sgRNA-mediated targeted gene modification method has been shown to be effective in inducing targeted gene mutations in Arabidopsis and other plant systems [32]. This method relies on expression of the nuclease domain containing Cas9 protein and a single-stranded guide RNA (sgRNA) that confers DNA target specificity. Binary vectors expressing both Cas 9 and a sgRNA have been developed and successfully transformed into Arabidopsis using a standard floral dip method [33]. Importantly, the mutations can be stably inherited and the process appears specific as other genes are not mutated [34]. However, if desired, careful selection of the sgRNA allows for the selective targeting of multiple genes containing identical stretches of sequence $[35,36]$. Thus, given the high degree of similarity between all three Camelina sub-genomes, in most cases it should be possible to simultaneously mutate all three homeologues of a specific gene.

With the demonstrated effectiveness of RNAi and the promise of CRISP/Cas9 genome editing, altering endogenous lipid biosynthetic fluxes can now be achieved seemingly at will. Thus mutants analogous to those in Arabidopsis should be easily generated, further facilitating the use of Camelina as an ideal oil seed metabolic 
engineering platform. Before discussing specific examples of Camelina metabolic engineering, a brief overview of fatty acid and TAG biosynthesis is presented.

\section{TAG Biosynthesis in Plants}

TAGs are the major seed storage lipids of most plants and are used to provide energy and carbon during germination. The overall pathway for the synthesis of TAG in the seeds of higher plants is complex (Figure 2); for a more detailed description of this important process, readers are encouraged to refer to recent and more in-depth reviews $[2,37,38]$.

\subsection{Fatty acid synthesis}

Briefly, fatty acids are synthesized in the plastids by a type II fatty acid synthase complex similar to that found in prokaryotes [39]. A repeated series of condensation, reduction and dehydration reactions add two carbon units to the elongating fatty acid chain, which is connected to an acyl carrier protein (ACP). Typically, fatty acids up to 16 or 18 carbons in length are synthesized, though shorter molecules are possible in other species. Introduction of a double bond through the action of a $\Delta 9$-desaturase can also occur in the plastid. Acyl-ACP thioesterases are responsible for the removal of the growing fatty acid from ACP. Of the two types of thioesterases, FatA enzymes preferentially remove oleate (18:1) whereas FatB thioesterases tend to remove shorter, saturated fatty acids. The fatty acids synthesized in the plastid are exported to the cytosol and converted to CoA forms, which can be used as acyl donor substrates by acyltransferases.

\subsection{Incorporation of fatty acids into TAGs}

Newly synthesized fatty acids are rapidly incorporated into phosphatidylcholine (PC) $[40,41]$. There they can be further modified by additional desaturation or the incorporation of other functional groups. An acyl editing pathway cycles fatty acids between PC and the acyl-CoA pool without the net synthesis of PC. Recent work has suggested that the movement of fatty acids both into and out of the PC pool is mediated by the forward and reverse reactions of lysophosphatidylcholine acyltransferases (LPCAT) [42-44]. Incorporation of fatty acids into TAG occurs via the Kennedy Pathway [45], which starts with sequential acylation of glycerol-3phosphate by glycerol-3-phosphate acyltransferases (GPATs) and lysophosphatidic acid acyltransferases (LPAATs) using acyl-CoA to produce phosphatidic acid (PA). PA is then dephosphorylated by PA phosphatases to create de novo diacylglycerol (DAG). DAG can then be used as a substrate in two different acyltransferase reactions that synthesize TAG. Diacylglycerol acyltransferases (DGAT) transfer the acyl group from acyl-CoAs to the $s n-3$ position of DAG to produce TAG [46]. Alternatively, phospholipid:diacylglycerol acyltransferases (PDAT) transfer the sn-2 acyl group from phospholipids to DAG to form TAG $[47,48]$. In Arabidopsis, DGAT1 is responsible for the synthesis of the majority of TAG [49], but in other species, such as flax, PDAT activity also plays an important role [50].

In addition to the de novo DAG pool formed via the Kennedy Pathway, labeling experiments have suggested the existence of a second, PC-derived DAG pool [40, 41]. In Arabidopsis, phosphatidylcholine: diacylglycerol 
cholinephosphotransferase (PDCT), which transfers the phosphocholine headgroup from PC to DAG, appears to be responsible for the bulk of the interconversion between PC and DAG [51]. However, other mechanisms to convert PC to DAG, such as the action of a phospholipase $\mathrm{C}$ or the reverse reaction of CDPcholine:diacylglycerol cholinephosphotransferase (CPT), are also possible [52]. This PC-derived DAG pool can then converted to TAG via DGAT or PDAT activity.

\subsection{Production of TAG containing unusual fatty acids in Arabidopsis}

A large number of plants also contain unusual fatty acid in TAGs [1]. These FA are typically derived from the further modification of fatty acids on PC molecules. Interestingly, many of the enzymes that catalyze these modifications like hydroxylation or epoxylation are variants of the membrane-bound desaturases responsible for the synthesis of polyunsaturated fatty acids [53]. One of the most highly studied unusual fatty acids is ricinoleic acid, a hydroxylated fatty acid which accounts for up to $90 \%$ of the fatty acids found in castor oil [1]. Ricinoleic acid is synthesized from oleic acid esterified to the $s n-2$ position of PC by the castor oleate $\Delta$-12 hydroxylase $(R c$ FAH12) [54, 55]. However, expression of $R c$ FAH12 in Arabidopsis led to seed oil containing relatively low levels (around 17\%) of hydroxy fatty acids [56]. Similar low results were also noted in efforts to engineer the synthesis of other unusual fatty acids in Arabidopsis [57]. These low levels of modified fatty acids in the seeds of transgenic plants appear to be caused by their inefficient removal from the site of their synthesis on PC and subsequent incorporation into TAG $[40,58,59]$. Indeed, the coexpression of castor derived enzymes with higher specificity for hydroxy fatty acid containing substrates increased the accumulation of these unusual fatty acids in the transgenic seed oil. For example, expression of a castor DGAT2 ortholog (RcDGAT2) along with RcFAH12 increased hydroxy fatty acid levels from $17 \%$ to $28 \%$ [60]. Likewise, coexpression of a castor PDAT led to similar sized increases [59]. Stacking these two acyltransferases with RcFAH12 only led to a small, but significant, further increase over either acyltransferase alone [59]. Finally, the addition of PDCT cloned from castor increased hydroxy fatty acids from $10 \%$ with only $C s$ FAH12 to 20\% [61]. An increased understanding of the flux of fatty acids from the site of synthesis to incorporation will lead to even higher increases of industrially useful fatty acids in the future.

\section{Synthesis of unusual lipids in Camelina}

Much of what we have learned from engineering oil seed metabolism in Arabidopsis has translated remarkably well to Camelina, not only with regard to hydroxy fatty acids but also with the production of omega-7 fatty acids and acetyl-TAGs.

\subsection{Production of high levels of hydroxy fatty acids in Camelina}

Transformation of Camelina with RcFAH12 under control of the seed specific phaseolin promoter yield lines capable of accumulating just over $6 \%$ ricinoleic acid along with smaller quantities of other hydroxy fatty acids [27]. Combining the expression of RcFAH12 with a fatty acid condensing enzyme from Physaria fendleri ( $L f K C S$ ), responsible for the elongation of hydroxy fatty acids, increased the 
accumulation of the elongated hydroxy fatty acids such as lesquerelic acid to $8 \%$ [62]. Interestingly, these seeds also accumulated slightly higher levels of ricinoleic acid leading to an overall increase in total hydroxy fatty acids from an average of $14 \%$ with $R c$ FAH alone to $19 \%$ with the addition of $L f K C S$. The increase was attributed to a more efficient removal of the elongated hydroxy fatty acids from PC, thus helping overcome the bottleneck in this process. Presumably, coexpression of castor acyltransferases in Camelina will lead to similar increases to hydroxyl fatty acids, similar to what was observed in Arabidopsis.

\subsection{Production of omega-7 unsaturated fatty acids}

Omega-7 unsaturated fatty acids such as palmitoleic acid (16:1 $\Delta 9)$ and vaccenic acid (18:1 111$)$ possess a number of physical properties that make them useful for industrial and biofuel applications. For example, it is predicted that shorter, monounsaturated fatty acids such as palmitoleic acid contain the optimal balance of chain length and double bonds to allow improved biodiesel cold-temperature properties and reduced $\mathrm{NO}_{\mathrm{x}}$ emissions while maintaining ignition quality and oxidative stability [63]. Omega-7 fatty acids can also be used as a source of valuable chemical precursors for industrial applications. In particular, the location of the single double bond in these fatty acids allows them to be used for the metathesis based production of 1-octene, a monomer in the synthesis of polyethylene.

Accumulation of high levels of omega-7 fatty acids was achieved in transgenic Arabidopsis seeds through the expression of a $\triangle 9$-18:0-ACP desaturase engineered with enhanced specificity towards 16:0-ACP and an extraplastidial $\Delta 9$ 16:0-CoA desaturase. Additionally, the activity of KASII which also uses 16:0-ACP as a substrate was suppressed and the elongation of $16: 1 \Delta 9$ was prevented by using an fae1 mutant background. Through this expression of exogenous genes combined with the elimination of competing pathways, omega- 7 fatty acid levels as high as 67 mol\% were achieved, essentially the same as the levels achieved in seeds that naturally accumulate these unusual fatty acids [64].

A similar strategy was subsequently employed in Camelina. However, instead of using mutant backgrounds, RNAi was used to suppress expression of the endogenous KASII and FAE1 genes. This suppression, combined with the expression of the same substrate-optimized $\triangle 9-18: 0$-ACP desaturase as before and a $\Delta 9-16: 0$ CoA desaturase from C. elegans, led to total omega-7 fatty acid levels of $44 \mathrm{~mol} \%$ [65]. Retransformation of this line with another copy of the $\Delta 9-18: 0-A C P$ desaturase and RNAi against FatB expression resulted in omega- 7 fatty acids levels of $66 \%$, one of the highest levels of unusual fatty acids synthesized in a transgenic oil seed [65]. Initial data from greenhouse-grown plants suggest that this high accumulation of omega-7 fatty acids did not affect the oil content, seed weight or germination efficiency [64]; future work will confirm whether these results translate under field conditions.

\subsection{Production of high levels of acetyl-TAGs in Camelina}

The seeds of Euonymus alatus (Burning Bush) and a few other plant species synthesize 3-acetyl-1,2-diacyl-sn-glycerols (acetyl-TAGs), unusual TAGs with an $s n$-3 acetate group $[66,67]$. Acetyl-TAGs possess different physical properties compared 
to regular seed oils, making them potentially useful for a number of different applications. For example, acetyl-TAGs possess a kinematic viscosity that is approximately $40 \%$ lower than that of typical TAGs [68]. As the high viscosity of vegetable oils prevents their direct use in standard diesel engines [69], one potential application for acetyl-TAGs is as an improved low-viscosity straight vegetable oil (SVO) biofuel. Indeed, acetyl-TAGs possess a viscosity in the range of Diesel \#4, a heavier grade diesel used in the constant speed engines found in locomotives, ships and heavy generators. Further, acetyl-TAGs also possess improved cold temperature properties compared to regular TAGs $[70,71]$, an important consideration, especially in colder climates. In addition to being used as a fuel, these properties of acetyl-TAGs lend themselves to other useful applications such as biodegradable lubricants, transformer oils and other products.

The gene encoding the diacylglycerol acetyltransferase (DAcT) activity required for the synthesis of acetyl-TAGs in Euonymus alatus (Burning Bush) was cloned using a comparative transcriptomic approach [68]. Expression of EaDAcT in wild-type Arabidopsis seeds resulted in the accumulation of up to $45 \mathrm{~mol} \%$ of acetyl-TAGs in the transgenic seed oil. Similar results were obtained in Camelina where lines transformed with EaDAcT accumulating on average 55 mol\% acetylTAGs [70]. Because EaDAcT uses the same DAG substrate as the endogenous DGAT1 and PDAT enzymes responsible for the synthesis of regular triacylglycerols, the elimination of this competing pathway represents one approach to increasing acetyl-TAG levels. Indeed, expression of EaDAcT in the Arabidopsis dgat1 background led to acetyl-TAG levels of $60-65 \mathrm{~mol} \%$ in the highest accumulating lines. Further, this result could be replicated in Camelina: EaDAcT expression combined with the RNAi-mediated suppression of the three Camelina DGAT1 homeologues led to transgenic lines capable of synthesizing up to $85 \mathrm{~mol} \%$ acetylTAGs [70]. Further analysis of the high acetyl-TAG lines revealed that the trait was stable through multiple generations. The average seed mass was not altered relative to wild-type controls and seed oil content was reduced only slightly. Importantly, germination rates of the seeds accumulating high levels of acetyl-TAGs were not greatly different from wild-type seed. These results stand in contrast to the synthesis of other unusual lipids in transgenic seeds where major reductions in seed size, oil content and germination were noted $[58,59,72,73]$. Typically these problems are associated with bottlenecks in the flux of unusual fatty acids from the PC pool where they are synthesized [74]. The synthesis of acetyl-TAGs avoids such constraints because EaDAcT functions at the end of a biosynthetic pathway and uses the readily available acetyl-CoA as a direct substrate. Further, because it appears that germinating seedlings can metabolize acetyl-TAGs as well as the regular TAGs stored in a seed [70], the complete replacement of regular TAG storage reserved with these unusually structured molecules should not pose a problem during the germination process.

\subsubsection{Enhancing the functionality of acetyl-TAGs}

The fatty acid composition of the acetyl-TAGs produced in different transgenic plant species is reflective of DAG molecular species found in those plants. By altering the composition of the DAG pool it should therefore be possible to incorporate different 
fatty acids at the $s n-1 / 2$ positions of acetyl-TAGs and further modulate the properties of these unusual lipids.

This scenario has already been successfully demonstrated with the production of high-oleic acetyl-TAGs [71]. High oleic Camelina lines generated using RNAi against CsFAD2 and CsFAE1 [28] were transformed with EaDAcT and RNAi against DGAT1 and PDAT. The resultant lines produced up to 70 mol\% acetyl-TAGs. Further, 3-acetyl-1,2-dioleoyl-sn-glycerol was the most abundant acetyl-TAG molecular species, comprising $47 \%$ of all acetyl-TAG molecular species [71]. The high proportion of oleic acid in the $s n-1$ and $s n-2$ positions of the acetyl-TAGs changed the physical properties of these molecules. For example, high-oleic acetylTAGs possessed a high oxidative stability index [75] reflective of the replacement of polyunsaturated fatty acids with the more stable mono-unsaturated oleic acid.

The ability to incorporate unusual fatty acids into acetyl-TAGs therefore allows a combinatorial approach in order to achieve a wider range of molecules with desired properties and increased value. For example, because viscosity appears to primarily depend on molecular weight [76], it is tempting to speculate that the incorporation of medium chain fatty acids at $s n-1 / 2$ will lead to further reductions in the viscosity of acetyl-TAGs. In vitro experiments suggest that EaDAcT is capable of acetylating diacylglycerol molecules with fatty acids as short as caproic acid [68] suggesting that the synthesis of acetyl-TAGs with a wide variety of fatty acids at sn$1 / 2$ is not excluded from a substrate specificity point of view.

\subsection{Functional testing of modified oil from transgenic Camelina}

The larger seeds of Camelina compared to Arabidopsis offer an obvious advantage in terms of producing larger quantities of seed oil for physical and chemical property testing. Thus, analysis of Camelina oil using differential scanning calorimetry (DSC) containing high levels of omega-7 fatty acids revealed alterations in thermal properties, such as a lower crystallization onset temperature, compared to seed oil from wild-type plants [65]. Similar analyses revealed that acetyl-TAGs possess improved cold-temperature properties, with crystallization of the $\alpha$ polymorphic form occurring $18^{\circ} \mathrm{C}$ lower than typical triacylglycerols [70]. Further, the ability to grown Camelina in the field allows for the generation of large quantities of modified oil seeds, permitting analyses that require larger amounts of material. For example, the standard tests for quantifying the cloud point or the pour point of a fuel each require $40 \mathrm{ml}$ of sample $[77,78]$. Other, more elaborate analyses such as cetane number determination consume up to $1 \mathrm{~L}$ of the test fuel [79]. Thus, the field growth of high acetyl-TAG Camelina lines allowed the generation of large quantities of modified oil, allowing the demonstration that transgenically produced acetyl-TAGs also possess a 40\% reduction in kinematic viscosity compared to regular seed oil [70]. Interestingly, high-oleic acetyl-TAGs possessed a higher viscosity than acetyl-TAGs synthesized in a wild-type Camelina background, probably due to the reduced levels of polyunsaturated fatty acid levels in the higholeic acetyl-TAGs [71]. Such physical property information is particularly valuable as it suggests that additional factors beyond molecular weight are important for viscosity. Importantly, insights such as this would not have been achievable without 
the ability to specifically engineer a designed oil trait in Camelina and then obtain large quantities of the resulting oil.

\section{Future Directions}

While Camelina already possesses many positive agronomic traits, it has not been the subject of much breeding, suggesting that further improvements in these characteristics are still possible. For example, increasing yield and seed oil content will enhance the competitiveness of Camelina compared to other oil seed crops. Improving tolerance to heat and other abiotic stresses will increase options for growth, both geographically and with regard to seasonal timing. A number of studies surveying different Camelina genotypes have demonstrated significant genetic variation in a number of important traits [13, 80-82], providing a basis for future breeding programs. However, the undifferentiated allohexaploid nature of the Camelina genome with multiple homeologues expressed will mean a complex mode of inheritance for various phenotypes, complicate breeding efforts. In this regard, the application of next generation sequencing technologies to develop dense marker maps and rapidly genotype individuals should allow the implementation of genomic selection models to speed up the breeding process [83].

\section{Conclusion}

Based on its ease of transformation and knowledge of its lipid biosynthetic pathways, Arabidopsis has long been used as the preferred model system with which to engineer the production of modified lipids. In addition to the examples that we detail, the production of many other unusual lipids, such as wax esters and epoxy-fatty acids, has been demonstrated in Arabidopsis [84, 85]. However, the development of an effective transformation method has allowed transgenic Camelina lines to be developed almost as easily as with Arabidopsis. Further, by allowing the manipulation of endogenous pathways, RNAi-mediated suppression and the potential of genome-editing techniques has effectively compensated for the lack of Camelina lipid biosynthetic mutants. The ease therefore of manipulating lipid composition, combined with the ability to grow Camelina in the field, make it an ideal platform to develop industrial lipids in transgenic oil seed crops. While this review has focused on the production of industrial lipids, it is worthwhile noting that Camelina has recently also been used for the production of nutritionally valuable omega-3 fatty acids $[86,87]$. This work involved the expression of multiple desaturase and elongase genes to successfully reconstitute eicosapentaenoic acid (20:5) or docosahexaenoic acid (22:6) biosynthetic pathways. Likewise, Camelina has also been used as a platform for the synthesis of the polymer poly-3hydroxybutyrate (PHB) [88]. It is therefore tempting to speculate that much future seed metabolic engineering will be performed in Camelina rather than Arabidopsis, allowing for more rapid introduction of plants capable of producing industrial seed oils into the field.

\section{Acknowledgements}


Research in the authors' laboratory focused on developing Camelina as a source of renewable fuel and materials is supported by grants from the U.S. Department of Energy under Award Number DE-SC0012459 and from the U.S. Department of Agriculture under Award Number 2015-67013-22815. This is contribution number 15-326-J from the Kansas Agricultural Experiment Station. 


\section{References}

1. Badami RC, Patil KB. (1980). Structure and occurrence of unusual fatty acids in minor seed oils. Prog Lipid Res. 19:119-153.

2. Li-Beisson Y, Shorrosh B, Beisson F, Andersson MX, Arondel V, Bates PD, Baud S, Bird D, DeBono A, Durrett TP, Franke RB, Graham IA, Katayama K, Kelly AA, Larson T, Markham JE, Miquel M, Molina I, Nishida I, Rowland O, Samuels L, Schmid KM, Wada H, Welti R, Xu C, Zallot R, Ohlrogge J. (2013). Acyl-Lipid Metabolism. The Arabidopsis Book. 11:e0161.

3. Wallis JG, Browse J. (2010). Lipid biochemists salute the genome. Plant J. 61:10921106.

4. Knorzer KH. (1978). Evolution and Spreading of Gold of Pleasure (CamelinaSativa S1). Ber Deut Bot Ges. 91:187-195.

5. Soriano N, Jr., Narani A. (2012). Evaluation of Biodiesel Derived from Camelina sativa Oil. J Am Oil Chem Soc. 89:917-923.

6. Putnam D, Budin J, Field L, Breene W. Camelina: a promising low-input oilseed. In: Janick J, Simon J, editors. New crops. New York, NY: John Wiley \& Sons; 1993. p. 314322.

7. Blackshaw R, Johnson E, Gan Y, May W, McAndrew D, Barthet V, McDonald T, Wispinski D. (2011). Alternative oilseed crops for biodiesel feedstock on the Canadian prairies. Can J Plant Sci. 91:889-896.

8. Dobre P, Jurcoane S. (2011). Camelina crop-opportunities for a sustainable agriculture. Scientific Papers-Series A, Agronomy. 54:420-424.

9. Zubr J. (1997). Oil-seed crop: Camelina sativa. Ind Crops Prod. 6:113-119. 10. Urbaniak SD, Caldwell CD, Zheljazkov VD, Lada R, Luan L. (2008). The effect of cultivar and applied nitrogen on the performance of Camelina sativa L. in the Maritime Provinces of Canada. Can J Plant Sci. 88:111-119.

11. Karcauskiene D, Sendzikiene E, Makareviciene V, Zaleckas E, Repsiene R, Ambrazaitiene D. (2014). False flax (Camelina sativa L.) as an alternative source for biodiesel production. Zemdirbyste. 101:161-168.

12. Wysocki DJ, Chastain TG, Schillinger WF, Guy SO, Karow RS. (2013). Camelina:

Seed yield response to applied nitrogen and sulfur. Field Crops Res. 145:60-66.

13. Enjalbert JN, Zheng SS, Johnson JJ, Mullen JL, Byrne PF, McKay JK. (2013).

Brassicaceae germplasm diversity for agronomic and seed quality traits under drought stress. Ind Crop Prod. 47:176-185.

14. Gugel RK, Falk KC. (2006). Agronomic and seed quality evaluation of Camelina sativa in western Canada. Can J Plant Sci. 86:1047-1058.

15. Hunsaker DJ, French AN, Clarke TR, El-Shikha DM. (2011). Water use, crop coefficients, and irrigation management criteria for camelina production in arid regions. Irrig Sci. 29:27-43.

16. Conn KL, Tewari JP, Dahiya JS. (1988). Resistance to Alternaria brassicae and phytoalexin-elicitation in rapeseed and other crucifers. Plant Sci. 56:21-25.

17. Sharma G, Dinesh Kumar V, Haque A, Bhat SR, Prakash S, Chopra VL. (2002). Brassica coenospecies: a rich reservoir for genetic resistance to leaf spot caused by Alternaria brassicae. Euphytica. 125:411-417. 
18. Li H, Barbetti MJ, Sivasithamparam K. (2005). Hazard from reliance on cruciferous hosts as sources of major gene-based resistance for managing blackleg (Leptosphaeria maculans) disease. Field Crop Res. 91:185-198.

19. Séguin-Swartz G, Eynck C, Gugel RK, Strelkov SE, Olivier CY, Li JL, Klein-Gebbinck H, Borhan H, Caldwell CD, Falk KC. (2009). Diseases of Camelina sativa (false flax). Can J Plant Pathol. 31:375-386.

20. Behera SK, Srivastava P, Tripathi R, Singh JP, Singh N. (2010). Evaluation of plant performance of Jatropha curcas L. under different agro-practices for optimizing biomass - A case study. Biomass Bioenerg. 34:30-41.

21. Shonnard DR, Williams L, Kalnes TN. (2010). Camelina-derived jet fuel and diesel: Sustainable advanced biofuels. Environ Prog Sustain Energy. 29:382-392. 22. Gesch RW, Archer DW. (2013). Double-cropping with winter camelina in the northern Corn Belt to produce fuel and food. Ind Crop Prod. 44:718-725.

23. Gesch RW, Archer DW, Berti MT. (2014). Dual Cropping Winter Camelina with Soybean in the Northern Corn Belt. Agron J. 106:1735-1745.

24. Krohn BJ, Fripp M. (2012). A life cycle assessment of biodiesel derived from the "niche filling" energy crop camelina in the USA. Appl Energ. 92:92-98.

25. Li X, Mupondwa E. (2014). Life cycle assessment of camelina oil derived biodiesel and jet fuel in the Canadian Prairies. Sci Total Environ. 481:17-26. 26. Liu X, Brost J, Hutcheon C, Guilfoil R, Wilson A, Leung S, Shewmaker C, Rooke S, Nguyen T, Kiser J, De Rocher J. (2012). Transformation of the oilseed crop Camelina sativa by Agrobacterium-mediated floral dip and simple large-scale screening of transformants. In Vitro Cell Dev Biol - Plant. 48:462-468.

27. Lu C, Kang J. (2008). Generation of transgenic plants of a potential oilseed crop Camelina sativa by Agrobacterium-mediated transformation. Plant Cell Rep. 27:273278.

28. Nguyen HT, Silva JE, Podicheti R, Macrander J, Yang W, Nazarenus TJ, Nam J-W, Jaworski JG, Lu C, Scheffler BE, Mockaitis K, Cahoon EB. (2013). Camelina seed transcriptome: a tool for meal and oil improvement and translational research. Plant Biotechnol J. 11:759-769.

29. Kagale S, Koh C, Nixon J, Bollina V, Clarke WE, Tuteja R, Spillane C, Robinson SJ, Links MG, Clarke C, Higgins EE, Huebert T, Sharpe AG, Parkin IAP. (2014). The emerging biofuel crop Camelina sativa retains a highly undifferentiated hexaploid genome structure. Nat Commun. 5:3706.

30. Hutcheon C, Ditt R, Beilstein M, Comai L, Schroeder J, Goldstein E, Shewmaker C, Nguyen T, De Rocher J, Kiser J. (2010). Polyploid genome of Camelina sativa revealed by isolation of fatty acid synthesis genes. BMC Plant Biol. 10:233.

31. Kang J, Snapp AR, Lu C. (2011). Identification of three genes encoding microsomal oleate desaturases (FAD2) from the oilseed crop Camelina sativa. Plant Physiol Biochem. 49:223-229.

32. Belhaj K, Chaparro-Garcia A, Kamoun S, Nekrasov V. (2013). Plant genome editing made easy: targeted mutagenesis in model and crop plants using the CRISPR/Cas system. Plant methods. 9:39.

33. Feng Z, Zhang B, Ding W, Liu X, Yang D-L, Wei P, Cao F, Zhu S, Zhang F, Mao Y, Zhu J-K. (2013). Efficient genome editing in plants using a CRISPR/Cas system. Cell Res. 23:1229-1232. 
34. Feng Z, Mao Y, Xu N, Zhang B, Wei P, Yang D-L, Wang Z, Zhang Z, Zheng R, Yang L, Zeng L, Liu X, Zhu J-K. (2014). Multigeneration analysis reveals the inheritance, specificity, and patterns of CRISPR/Cas-induced gene modifications in Arabidopsis. Proc Natl Acad Sci USA. 111:4632-4637.

35. Li J-F, Norville JE, Aach J, McCormack M, Zhang D, Bush J, Church GM, Sheen J. (2013). Multiplex and homologous recombination-mediated genome editing in Arabidopsis and Nicotiana benthamiana using guide RNA and Cas9. Nat Biotech. 31:688-691.

36. Mao Y, Zhang H, Xu N, Zhang B, Gou F, Zhu J-K. (2013). Application of the CRISPR-Cas System for Efficient Genome Engineering in Plants. Mol Plant. 6:20082011.

37. Bates PD, Browse J. (2012). The significance of different diacylgycerol synthesis pathways on plant oil composition and bioengineering. Frontiers Plant Sci. 3. 38. Bates PD, Stymne S, Ohlrogge J. (2013). Biochemical pathways in seed oil synthesis. Curr Opinion Plant Biol. 16:358-364.

39. Ohlrogge JB, Jaworski JG. (1997). Regulation of Fatty Acid Synthesis. 48:109-136. 40. Bates PD, Browse J. (2011). The pathway of triacylglycerol synthesis through phosphatidylcholine in Arabidopsis produces a bottleneck for the accumulation of unusual fatty acids in transgenic seeds. Plant J. 68:387-399.

41. Bates PD, Durrett TP, Ohlrogge JB, Pollard M. (2009). Analysis of acyl fluxes through multiple pathways of triacylglycerol synthesis in developing soybean embryos. Plant Physiol. 150:55-72.

42. Bates PD, Fatihi A, Snapp AR, Carlsson AS, Browse J, Lu C. (2012). Acyl Editing and Headgroup Exchange Are the Major Mechanisms That Direct Polyunsaturated Fatty Acid Flux into Triacylglycerols. Plant Physiol. 160:1530-1539.

43. Lager I, Yilmaz JL, Zhou X-R, Jasieniecka K, Kazachkov M, Wang P, Zou J, Weselake R, Smith MA, Bayon S, Dyer JM, Shockey JM, Heinz E, Green A, Banas A, Stymne S. (2013). Plant Acyl-CoA:Lysophosphatidylcholine Acyltransferases (LPCATs) Have Different Specificities in Their Forward and Reverse Reactions. J Biol Chem. 288:36902-36914.

44. Wang L, Shen W, Kazachkov M, Chen G, Chen Q, Carlsson AS, Stymne S, Weselake RJ, Zou J. (2012). Metabolic Interactions between the Lands Cycle and the Kennedy Pathway of Glycerolipid Synthesis in Arabidopsis Developing Seeds. Plant Cell. 24:4652-4669.

45. Kennedy EP. (1961). Biosynthesis of complex lipids. Fed Proc. 20:934-940.

46. Liu Q, Siloto RMP, Lehner R, Stone SJ, Weselake RJ. (2012). Acyl-

CoA:diacylglycerol acyltransferase: Molecular biology, biochemistry and biotechnology. Prog Lipid Res. 51:350-377.

47. Dahlqvist A, Stahl U, Lenman M, Banas A, Lee M, Sandager L, Ronne H, Stymne H. (2000). Phospholipid : diacylglycerol acyltransferase: An enzyme that catalyzes the acyl-CoA-independent formation of triacylglycerol in yeast and plants. Proc Natl Acad Sci USA. 97:6487-6492.

48. Stahl U, Carlsson AS, Lenman M, Dahlqvist A, Huang B, Banas W, Banas A, Stymne S. (2004). Cloning and functional characterization of a phospholipid:diacylglycerol acyltransferase from Arabidopsis. Plant Physiol. 135:1324-1335. 
49. Zhang M, Fan J, Taylor DC, Ohlrogge JB. (2009). DGAT1 and PDAT1

Acyltransferases Have Overlapping Functions in Arabidopsis Triacylglycerol

Biosynthesis and Are Essential for Normal Pollen and Seed Development. Plant Cell.

21:3885-3901.

50. Pan X, Siloto RMP, Wickramarathna AD, Mietkiewska E, Weselake RJ. (2013).

Identification of a Pair of Phospholipid:Diacylglycerol Acyltransferases from

Developing Flax (Linum usitatissimum L.) Seed Catalyzing the Selective Production

of Trilinolenin. J Biol Chem. 288:24173-24188.

51. Lu C, Xin Z, Ren Z, Miquel M, Browse J. (2009). An enzyme regulating

triacylglycerol composition is encoded by the ROD1 gene of Arabidopsis. Proc Natl

Acad Sci USA. 106:18837-18842.

52. Slack CR, Campbell LC, Browse JA, Roughan PG. (1983). Some evidence for the

reversibility of the cholinephosphotransferase-catalyzed reaction in developing

linseed cotyledons in vivo. Biochim Biophys Acta. 754:10-20.

53. Broun P, Shanklin J, Whittle E, Somerville C. (1998). Catalytic Plasticity of Fatty Acid Modification Enzymes Underlying Chemical Diversity of Plant Lipids. Science. 282:1315-1317.

54. Bafor M, Smith MA, Jonsson L, Stobart K, Stymne S. (1991). Ricinoleic acid biosynthesis and triacylglycerol assembly in microsomal preparations from developing castor-bean (Ricinus communis) endosperm. Biochem J. 280:507-514. 55. van de Loo FJ, Broun P, Turner S, Somerville C. (1995). An oleate 12-hydroxylase from Ricinus communis L. is a fatty acyl desaturase homolog. Proc Natl Acad Sci U S A. 92:6743-6747.

56. Broun P, Somerville C. (1997). Accumulation of Ricinoleic, Lesquerolic, and Densipolic Acids in Seeds of Transgenic Arabidopsis Plants That Express a Fatty Acyl Hydroxylase cDNA from Castor Bean. Plant Physiol. 113:933-942.

57. Thelen JJ, Ohlrogge JB. (2002). Metabolic engineering of fatty acid biosynthesis in plants. Metab Eng. 4:12-21.

58. Cahoon EB, Dietrich CR, Meyer K, Damude HG, Dyer JM, Kinney AJ. (2006). Conjugated fatty acids accumulate to high levels in phospholipids of metabolically engineered soybean and Arabidopsis seeds. Phytochemistry. 67:1166-1176.

59. van Erp H, Bates PD, Burgal J, Shockey J, Browse J. (2011). Castor

Phospholipid:Diacylglycerol Acyltransferase Facilitates Efficient Metabolism of Hydroxy Fatty Acids in Transgenic Arabidopsis. Plant Physiol. 155:683-693.

60. Burgal J, Shockey J, Lu C, Dyer J, Larson T, Graham I, Browse J. (2008). Metabolic engineering of hydroxy fatty acid production in plants: RcDGAT2 drives dramatic increases in ricinoleate levels in seed oil. Plant Biotechnol J. 6:819-831.

61. Hu Z, Ren Z, Lu C. (2012). The Phosphatidylcholine Diacylglycerol

Cholinephosphotransferase Is Required for Efficient Hydroxy Fatty Acid

Accumulation in Transgenic Arabidopsis. Plant Physiol. 158:1944-1954.

62. Snapp AR, Kang J, Qi X, Lu C. (2014). A fatty acid condensing enzyme from

Physaria fendleri increases hydroxy fatty acid accumulation in transgenic oilseeds of Camelina sativa. Planta. 240:599-610.

63. Durrett TP, Benning C, Ohlrogge J. (2008). Plant triacylglycerols as feedstocks for the production of biofuels. Plant J. 54:593-607. 
64. Nguyen HT, Mishra G, Whittle E, Pidkowich MS, Bevan SA, Merlo AO, Walsh TA, Shanklin J. (2010). Metabolic Engineering of Seeds Can Achieve Levels of $\omega$-7 Fatty Acids Comparable with the Highest Levels Found in Natural Plant Sources. Plant Physiol. 154:1897-1904.

65. Nguyen HT, Park H, Koster KL, Cahoon RE, Nguyen HTM, Shanklin J, Clemente TE, Cahoon EB. (2015). Redirection of metabolic flux for high levels of omega-7 monounsaturated fatty acid accumulation in camelina seeds. Plant Biotechnol J. 13:38-50.

66. Bagby MO, Smith CR, Jr. (1967). Asymmetric triglycerides from Impatiens edgeworthii seed oil. Biochim Biophys Acta. 137:475-477.

67. Kleiman R, Miller RW, Earle FR, Wolff IA. (1967). (S)-1,2-diacyl-3-acetins: Optically active triglycerides from Euonymus verrucosus seed oil. Lipids. 2:473-478. 68. Durrett TP, McClosky DD, Tumaney AW, Elzinga DA, Ohlrogge J, Pollard M. (2010). A distinct DGAT with $s n-3$ acetyltransferase activity that synthesizes unusual, reduced-viscosity oils in Euonymus and transgenic seeds. Proc Natl Acad Sci USA. 107:9464-9469.

69. Ryan TW, Callahan TJ, Dodge LG. (1983). Characterization of Composition, Spray and Engine Combustion of 14 Vegetable Oils. J Am Oil Chem Soc. 60:736-736. 70. Liu J, Rice A, McGlew K, Shaw V, Park H, Clemente T, Pollard M, Ohlrogge J, Durrett TP. (2015). Metabolic engineering of oilseed crops top produce high levels of novel acetyl glyceride oils with reduced viscosity, freezing point and calorific value. Plant Biotechnol J.

71. Liu J, Tjellström H, McGlew K, Shaw V, Rice A, Simpson J, Kosma D, Ma W, Yang W, Strawsine M, Cahoon E, Durrett TP, Ohlrogge J. (2015). Field production, purification and analysis of high-oleic acetyl-triacylglycerols from transgenic Camelina sativa. Ind Crop Prod. 65:259-268.

72. Li R, Yu K, Wu Y, Tateno M, Hatanaka T, Hildebrand DF. (2012). Vernonia DGATs can complement the disrupted oil and protein metabolism in epoxygenaseexpressing soybean seeds. Metab Eng. 14:29-38.

73. Singh S, Thomaeus S, Lee M, Stymne S, Green A. (2001). Transgenic expression of a $\Delta 12$-epoxygenase gene in Arabidopsis seeds inhibits accumulation of linoleic acid. Planta. 212:872-879.

74. Bates PD, Johnson SR, Cao X, Li J, Nam J-W, Jaworski JG, Ohlrogge JB, Browse J. (2014). Fatty acid synthesis is inhibited by inefficient utilization of unusual fatty acids for glycerolipid assembly. Proc Natl Acad Sci USA. 111:1204-1209.

75. Knothe G, Dunn RO. (2003). Dependence of oil stability index of fatty compounds on their structure and concentration and presence of metals. 80:1021-1026.

76. Niedzielski EL. (1976). Neopentyl Polyol Ester Lubricants - Bulk Property Optimization. Ind Eng Chem Prod Res Dev. 15:54-58.

77. ASTM D97-12. Standard Test Method for Pour Point of Petroleum Products. ASTM International, West Conshohocken, PA. 2012.

78. ASTM D2500-11. Standard Test Method for Cloud Point of Petroleum Products. ASTM International, West Conshohocken, PA. 2011.

79. ASTM D613-14. Standard Test Method for Cetane Number of Diesel Fuel Oil. ASTM International, West Conshohocken, PA. 2014. 
80. Rodríguez-Rodríguez MF, Sánchez-García A, Salas JJ, Garcés R, Martínez-Force E. (2013). Characterization of the morphological changes and fatty acid profile of developing Camelina sativa seeds. Ind Crop Prod. 50:673-679.

81. Vollmann J, Grausgruber H, Stift G, Dryzhyruk V, Lelley T. (2005). Genetic diversity in camelina germplasm as revealed by seed quality characteristics and RAPD polymorphism. Plant Breed. 124:446-453.

82. Vollmann J, Moritz T, Kargl C, Baumgartner S, Wagentristl H. (2007). Agronomic evaluation of camelina genotypes selected for seed quality characteristics. Ind Crop Prod. 26:270-277.

83. Desta ZA, Ortiz R. (2014). Genomic selection: genome-wide prediction in plant improvement. Trends Plant Sci. 19:592-601.

84. Heilmann M, Iven T, Ahmann K, Hornung E, Stymne S, Feussner I. (2012).

Production of wax esters in plant seed oils by oleosomal cotargeting of biosynthetic enzymes. J Lipid Res. 53:2153-2161.

85. Zhou X, Singh S, Liu Q, Green A. (2006). Combined transgenic expression of $\Delta 12$ desaturase and $\Delta 12$-epoxygenase in high linoleic acid seeds leads to increased accumulation of vernolic acid. Funct Plant Biol. 33:585-592.

86. Ruiz-Lopez N, Haslam RP, Napier JA, Sayanova O. (2014). Successful high-level accumulation of fish oil omega-3 long-chain polyunsaturated fatty acids in a transgenic oilseed crop. Plant J. 77:198-208.

87. Ruiz-Lopez N, Haslam RP, Usher S, Napier JA, Sayanova O. (2015). An alternative pathway for the effective production of the omega-3 long-chain polyunsaturates EPA and ETA in transgenic oilseeds. Plant Biotechnol J. doi: 10.1111/pbi.12328 88. Malik MR, Yang W, Patterson N, Tang J, Wellinghoff RL, Preuss ML, Burkitt C, Sharma N, Ji Y, Jez JM, Peoples OP, Jaworski JG, Cahoon EB, Snell KD. (2015). Production of high levels of poly-3-hydroxybutyrate in plastids of Camelina sativa seeds. Plant Biotechnol J. 13:675-688. 
Figure 1. Fatty acid composition of seed oil from canola, soybean and Camelina. Values are from Putnam et al. (1993).

Figure 2. Overview of triacylglycerol biosynthesis in seeds. Dashed borders are used to indicate different metabolite pools within the endoplasmic reticulem. Compound abbreviations: ACP, acyl carrier protein; CoA, coenzyme A; DAG, diacylglycerol; DHAP, dihydroxyacetone phosphate; G-3-P, glycerol-3-phosphate; LPA, lysophosphatidic acid; LPC, lysophosphatidylcholine; PA, phosphatidic acid; PC, phosphotidylcholine; PUFA, polyunsaturated fatty acid; TAG, triacylglycerol Enzyme abbreviations: CPT, CDP-choline:diacylglycerol cholinephosphotransferase; $\mathrm{CPT}_{\mathrm{r}}$, reverse activity of CPT; DGAT, diacylglycerol acyltransferase; FAD, fatty acid desaturase; Fat, fatty acid thioesterase; GPAT, glycerol-3-phosphate acyltransferase; KAS, ketoacyl-ACP synthase; LPAAT, lysophosphatidic acid acyltransferase; LPCAT, lysophosphatidylcholine acyltransferase; PAP, phosphatidic acid phosphatase; PDAT, phosphatidylcholine: diacylglycerol acyltransferase; PDCT, phosphatidylcholine: diacylglycerol cholinephosphotransferase; PLC, phospholipase C; SAD, stearoyl desaturase. 


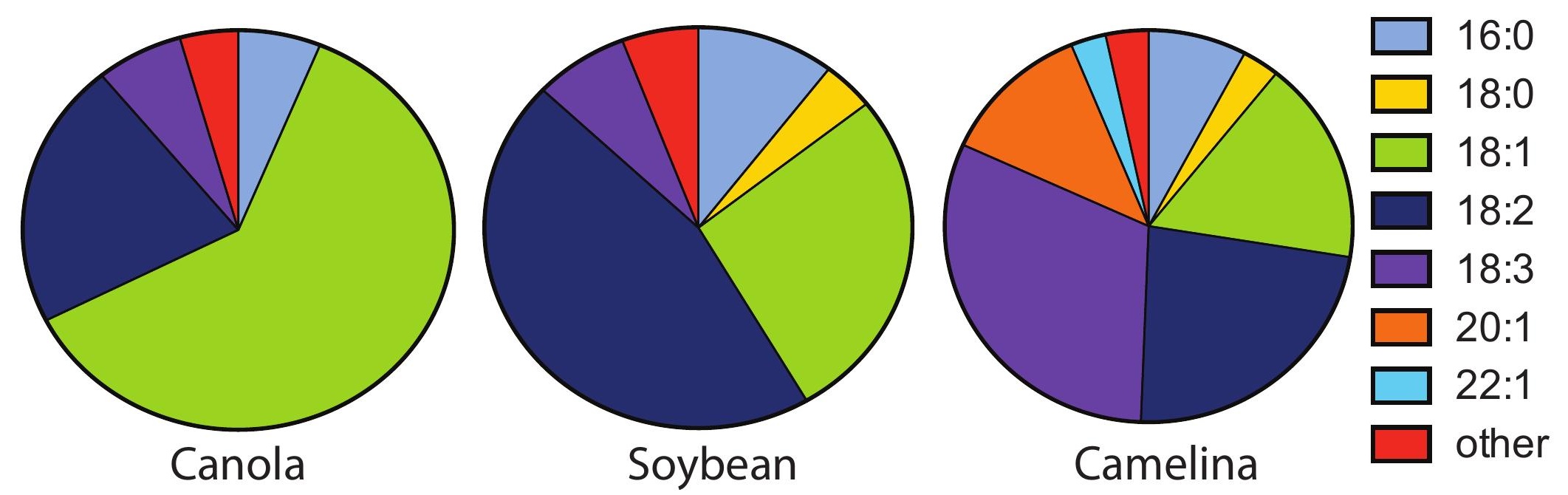

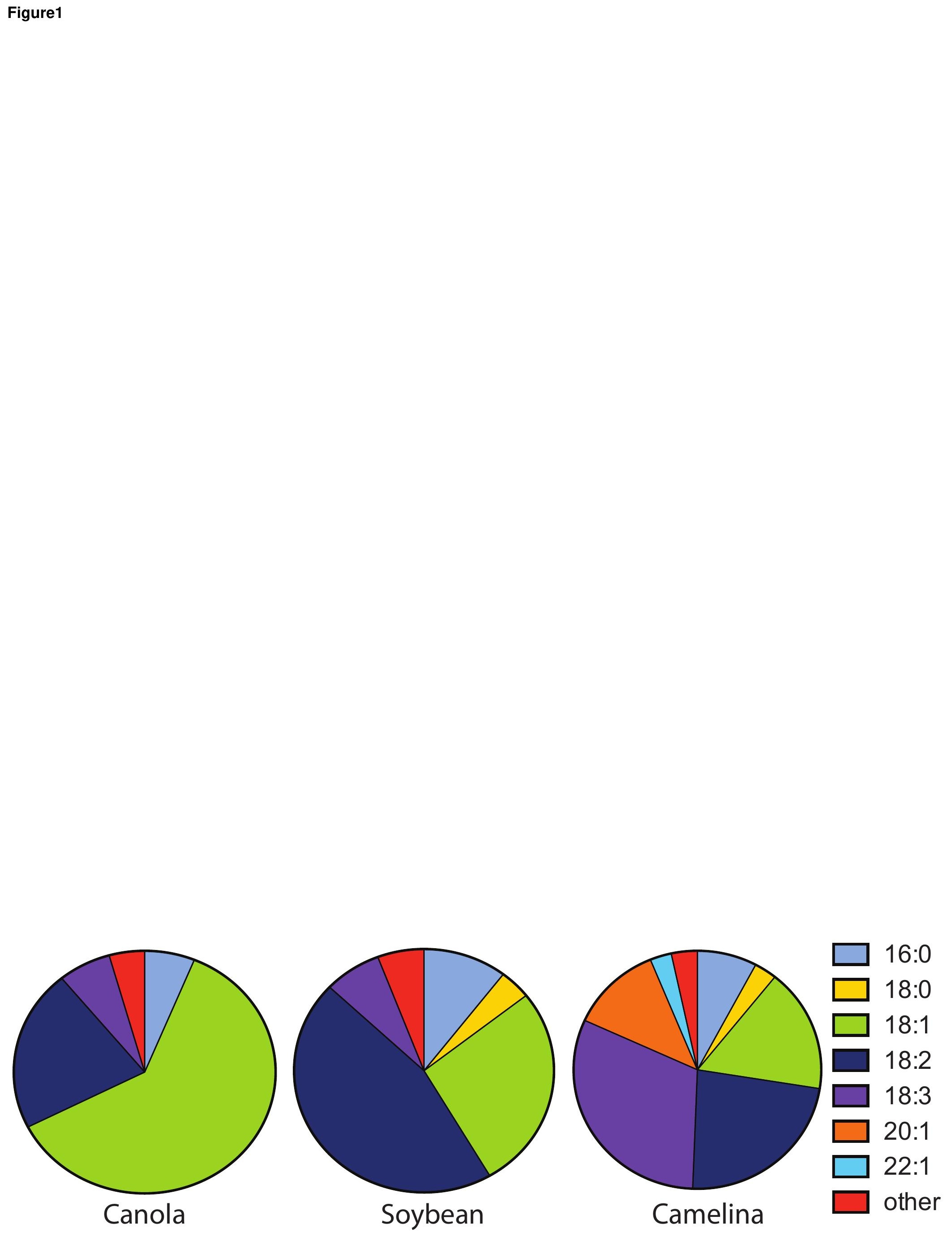


Plastid Acetyl-CoA Fatty acid synthesis

Fatty acid synthesis $16: 0-\mathrm{ACP} \stackrel{\text { KASII }}{\longrightarrow} 18: 0-\mathrm{ACP}$ SAD

Plastid Acetyl-COA $\longrightarrow$ 16:0-ACP $\longrightarrow$ 18:0-ACP $\longrightarrow$ 18:1-ACP

$\mathrm{DHAP} \longrightarrow \mathrm{G}-3-\mathrm{P}$

FatB

FatA

FatA

Endoplasmic reticulum
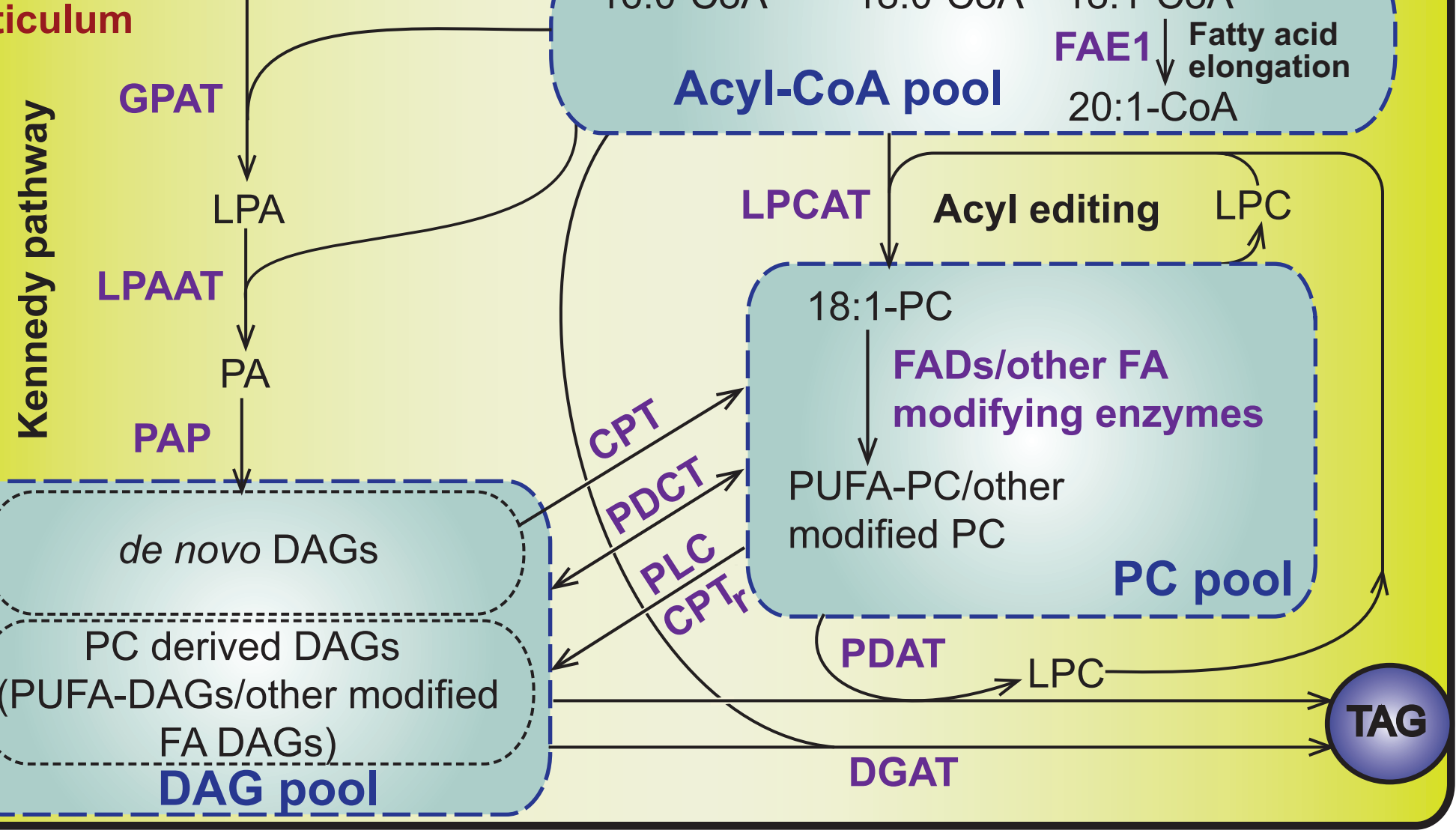